\title{
Von der Entgrenzung zur Eingrenzung: Krise und Wandel der europäischen Integration
}

\author{
Frank Schimmelfennig*
}

The "polycrisis" of the European Union (EU) presents integration research with major conceptual and theoretical challenges. In the interest of an adequate understanding of current developments in European integration, this contribution argues for opening the dominant concept of integration in order to systematically include the integration of the EU's external boundaries and to overcome its liberal bias. In this perspective, the current change in European integration consists mainly in external rebordering. It constitutes a correction of the pre-crisis type of debordering integration - a correction, which is conducive to the consolidation of European integration in principle.

Im zurückliegenden Jahrzehnt befand sich die Europäische Union (EU) im permanenten Krisenmodus. Zunächst schien die Ratifikation des Vertrags von Lissabon 2009 eine institutionelle Konsolidierung nach mühevollen Verhandlungen über die Verfassung der EU zu verheißen. Im gleichen Jahr markierten jedoch die wachsenden Zahlungsbilanzschwierigkeiten Griechenlands den Beginn der Eurokrise. Als diese im Sommer 2015 mit der letztlichen Zustimmung der griechischen Regierung zu den Konditionen des dritten griechischen Bail-outs überwunden war, signalisierte die sprunghafte Zunahme der Fluchtbewegungen über die Ägäis den Auftakt zur bis heute ungelösten Krise des Schengen-/DublinGrenz- und Asylregimes. Als die Flüchtlingszahlen im Laufe des Jahres 2016 wieder deutlich zurückgingen, stimmten die britischen WählerInnen für den Brexit und lösten damit erstmals in der Geschichte der Union Verhandlungen über den Austritt eines Mitgliedstaates aus der EU aus. Im gleichen Zeitraum entwickelte sich - vor allem in einigen ost- und mitteleuropäischen Mitgliedstaaten - schleichend eine Rechtsstaatlichkeitskrise, die 2017 dazu führte, dass - ebenfalls zum ersten Mal - Verfahren nach Art. 7 des Vertrags über die Europäische Union (EUV) wegen der Verletzung fundamentaler Werte der EU in die Wege geleitet wurden. Wachstum und Wahlerfolge populistischer Parteien begleiteten diese Krisen.

Im Vergleich zu den politischen Herausforderungen der aktuellen Krisenperiode verblassen die Ereignisse der bisher als größte Krisenphase der Integration gehandelten 1960er Jahre, die mit dem Namen des französischen Präsidenten Charles de Gaulle verbunden ist. War die frühere Krise vor allem eine Periode der Stagnation, in der beabsichtigte Integrationsschritte - wie die Norderweiterung oder der Übergang zu Mehrheitsentscheidungen im Rat der EU - blockiert wurden, so zeigen sich in der aktuellen Krise Desintegrationserscheinungen, die das bestehende Integrationsniveau infrage stellen.

Außerdem hält die gegenwärtige Krisenperiode erhebliche konzeptionelle und theoretische Herausforderungen für die Integrationsforschung bereit. War die Vorliebe der traditionellen Integrationstheorien für die Erklärung von Integrationsfortschritten bisher durch die Empirie weitgehend gedeckt, so zwingen die aktuellen Entwicklungen die Integrationsforschung dazu, Krisen- und Desintegrationsprozesse und ihre Bedingungen stärker in den

\footnotetext{
* Prof. Dr. Frank Schimmelfennig, Professor für Europäische Politik, ETH Zürich.
} 
Blick zu nehmen. Mit der Entstehung einer „postfunktionalistischen“ Alternative zu den traditionellen Integrationstheorien ${ }^{1}$ und einer Reihe von Neuerscheinungen, die Konzepte und theoretische Ansätze der Desintegration systematisch durchdeklinieren, befindet sich die Integrationsforschung in dieser Hinsicht bereits auf einem guten Weg. ${ }^{2}$ Außerdem zeigt sich, dass auch die traditionellen Integrationstheorien - Neofunktionalismus und Intergouvernementalismus - überzeugende Erklärungen für die „Polykrise“ der EU und ihre Ergebnisse anbieten. ${ }^{3}$

Ich argumentiere in diesem Beitrag, dass die Integrationsforschung allerdings unter einem doppelten konzeptionellen Bias leidet. Zum einen ist sie auf die interne Entgrenzung der EU, also die Öffnung und supranationale Kontrolle der Grenzen zwischen den Mitgliedstaaten, fokussiert und vernachlässigt dabei die zweite konstitutive Dimension der Integration: die externe Grenzziehung. Zum anderen hat sie die Tendenz, europäische Integration mit einer liberalen Programmatik gleichzusetzen.

Diese Verengung des Integrationskonzepts ist im Rückblick auf die dynamische Integrationsphase seit etwa der Mitte der 1980er Jahre nur zu verständlich. Diese Phase war durch weitreichende Fortschritte beim Abbau der internen Grenzen, ein liberales Integrationsparadigma und weitgehend unproblematische externe Grenzen geprägt. Jetzt verstellen das liberale Bias und der Fokus auf die internen Grenzen allerdings den Blick auf potenzielle Ursachen und Konsequenzen der Krisen. Zum einen wäre es falsch, eine Abkehr vom dominanten liberalen Integrationsparadigma der vergangenen Jahrzehnte pauschal als Desintegration zu disqualifizieren. Vielmehr wäre es wünschenswert, den Integrationsbegriff von programmatischen Festlegungen zu befreien. Zum anderen wäre es gewinnbringend, auch die Öffnung und Schließung der äußeren Grenzen der EU als eine zentrale Dimension der Integration zu begreifen und das Wechselverhältnis von innerer und äußerer Entgrenzung und Eingrenzung in die Analyse der Integrationsentwicklung einzubeziehen.

Eine solche Öffnung und Erweiterung des Integrationsbegriffs, so meine These, zeigt, dass die aktuellen Krisen der EU in erster Linie dem liberalen Integrationsparadigma und einer Grenzkonfiguration entspringen, die seit der Mitte der 1980er Jahre auf die gleichzeitige Öffnung der inneren und äußeren Grenzen der EU gesetzt hat. Ich behaupte weiterhin, dass wir aktuell eine partielle Abkehr von diesem liberalen Integrationsparadigma - nicht nur seitens der populistischen EU-Skeptiker, sondern auch im EU-freundlichen Mainstream - und eine zunehmende Schließung der äußeren Grenzen der EU beobachten können. Es wäre jedoch verkürzt, diese Entwicklung als Anzeichen einer europäischen Desintegration zu werten. Vielmehr handelt es sich um eine Abkehr von einem Integrationstyp, der auf die Kombination von externer und interner Entgrenzung setzt, und um eine Kurskorrektur zugunsten einer Integration, die externe Eingrenzung betreibt, um die interne

1 Liesbet Hooghe/Gary Marks: A Postfunctionalist Theory of European Integration: From Permissive Consensus to Constraining Dissensus, in: British Journal of Political Science 1/2009, S. $1 \mathrm{ff}$.

2 Vgl. vor allem Annegret Eppler/Henrik Scheller (Hrsg.): Zur Konzeptionalisierung europäischer Desintegration. Zug- und Gegenkräfte im europäischen Integrationsprozess, Baden-Baden 2013; Hans Vollaard: European Disintegration. A Search for Explanations, Basingstoke 2018.

3 Vgl. u.a. Demosthenes Ioannou/Patrick Leblond/Arne Niemann: European integration and the crisis: practice and theory, in: Journal of European Public Policy 2/2015, S. 155ff.; Arne Niemann/Natascha Zaun: EU Refugee Policies and Politics in Times of Crisis: Theoretical and Empirical Perspectives, in: Journal of Common Market Studies 1/2018, S. 3ff.; Liesbet Hooghe/Gary Marks: Grand theories of European integration in the twenty-first century, in: Journal of European Public Policy 8/2019, S. 1113ff. 
Entgrenzung zu bewahren - und damit die europäische Integration zu konsolidieren und zu stärken. Insgesamt führt die hier vorgeschlagene Konzeption also zu einer offeneren und weniger „pessimistischen“ Einschätzung der Entwicklung der europäischen Integration, als sie die postfunktionalistische und Desintegrationsliteratur gemeinhin vertreten.

Der Artikel gliedert sich in drei Teile. Zunächst werde ich diskutieren, wie die Integrationstheorie bisher auf die Krisen der europäischen Integration reagiert hat und wo aus meiner Sicht konzeptionelle Defizite bestehen. Danach werde ich die Öffnung und Erweiterung des Integrationskonzepts vorstellen, die ich in diesen einleitenden Überlegungen bereits skizziert habe, und im Anschluss an Stein Rokkan und Stefano Bartolini einige theoretische Erwartungen formulieren. Anschließend werde ich einen groben Überblick über die Integrationsentwicklung geben, um die erweiterte Konzeption der Integration und die theoretischen Erwartungen empirisch zu plausibilisieren.

\section{Integrationskrisen und Integrationstheorien}

Das Krisenjahrzehnt der EU ist nicht ohne Folgen für die Theorieentwicklung zur EU und zur europäischen Integration geblieben. Erstens führte es dazu, dass die Integrationstheorie an sich überhaupt erneut Aufmerksamkeit und Bedeutung gewann. Im Jahrzehnt davor hatte sich die Ansicht Bahn gebrochen, mit der EU sei in Maastricht ein in seinen wesentlichen Zügen stabiles konstitutionelles Arrangement entstanden ${ }^{4}$ - gewissermaßen ein „Maastricht im Dauerzustand“. ${ }^{5}$ Dementsprechend sei es gewinnbringender, die EU als ein politisches System unter anderen ${ }^{6}$ oder als ein besonderes System des MehrebenenRegierens $^{7} \mathrm{zu}$ verstehen und $\mathrm{zu}$ analysieren. An die Stelle der Integrationstheorien traten daher Konzepte und Ansätze aus der Vergleichenden Politikwissenschaft, der Policy-Analyse und der Föderalismusforschung. Die Krisen der EU mit ihren Integrationssprüngen (in der Eurozone) und Zerfallsprozessen (Flüchtlingskrise und Brexit) haben deutlich gemacht, dass das politische System der EU und seine konstitutionellen Rahmenbedingungen keineswegs so stabil sind wie angenommen. ${ }^{8}$

Zweitens führte die Krisenperiode zu einem bemerkenswerten Comeback der traditionellen Integrationstheorien. Der Liberale Intergouvernementalismus ${ }^{9}$ hat sich bei der Analyse der staatlichen Präferenzen, der intergouvernementalen und interinstitutionellen Verhandlungen sowie der materiellen und institutionellen Integrationsergebnisse in den Krisen als nach wie vor weitgehend erklärungskräftig erwiesen. ${ }^{10}$ Besonders vielversprechend

4 So z.B. Andrew Moravcsik: The European Constitutional Compromise and the neofunctionalist legacy, in: Journal of European Public Policy 2/2005, S. 349ff.

5 Markus Jachtenfuchs/Beate Kohler-Koch: Regieren im dynamischen Mehrebenensystem, in: Markus Jachtenfuchs/Beate Kohler-Koch (Hrsg.): Europäische Integration, Wiesbaden 1996, S. 15ff., hier S. 21.

6 Simon Hix: The Political System of the European Union, Basingstoke 1999.

7 Jachtenfuchs/Kohler-Koch: Regieren, 1996; Liesbet Hooghe/Gary Marks: Multi-Level Governance and European Integration, Lanham u.a. 2001.

8 Mareike Kleine/Mark Pollack: Liberal Intergovernmentalism and Its Critics, in: Journal of Common Market Studies 7/2018, S. 1493ff., hier S. 1503ff.

9 Andrew Moravcsik: The Choice for Europe. Social Purpose and State Power from Messina to Maastricht, Ithaca 1998.

10 Vgl. Felix Biermann et al.: Political (non-)reform in the euro crisis and the refugee crisis: a liberal intergovernmentalist explanation, in: Journal of European Public Policy 2/2018, S. 246ff.; Frank Schimmelfennig: Liberal Intergovernmentalism and the Crises of the European Union, in: Journal of Common Market Studies 7/2018, S. $1578 f f$. 
erscheint jedoch eine Synthese von Liberalem Intergouvernementalismus und Neofunktionalismus, die nationales Eigeninteresse und Verhandlungsmacht in harten zwischenstaatlichen Krisenverhandlungen (Intergouvernementalismus) mit der Pfadabhängigkeit der Integration (Neofunktionalismus) verbindet. Ob Integrationskrisen zu Integrationswachstum führen, hängt demnach von endogener Interdependenz und supranationaler Kapazität ab also davon, ob frühere Integrationsschritte zum einen eine so starke Interdependenz zwischen den Mitgliedstaaten erzeugen, dass nationale Alleingänge in der Krise zu kostspielig erscheinen, und zum anderen handlungsfähige supranationale Institutionen hervorbringen, die ein effektives Krisenmanagement betreiben können. ${ }^{11}$ Mit ihrem Konzept des „Failing Forward“ haben Erik Jones, Daniel Kelemen und Sophie Meunier für diese Theorieverbindung eine griffige Formel gefunden. ${ }^{12}$

Allerdings lassen diese Theorien keinen systematischen Platz für die wachsende innerstaatliche Politisierung der europäischen Integration und das Aufkommen populistischer Parteien und Regierungen. Sie ignorieren die Verschiebungen in der öffentlichen Meinung und im Parteienspektrum oder halten sie jedenfalls für nicht geeignet, die Ergebnisse der europäischen Krisenpolitik zu erklären. ${ }^{13}$ Zumindest beim Brexit stoßen sie damit jedoch an ihre Grenzen. ${ }^{14}$ Außerdem sind der Liberale Intergouvernementalismus und der Neofunktionalismus aufgrund ihres Entstehungskontexts in erster Linie Theorien des Integrationsfortschritts, die zu den Zerfallserscheinungen europäischer Integration wenig zu sagen haben. ${ }^{15}$

Das hat eine Reihe von Autoren dazu veranlasst, sich theoretisch mit „europäischer Desintegration" zu befassen. Zum einen haben sie die bestehenden Theorien gegen den Strich gebürstet, um zu sehen, was sie zur Erklärung von Rückbau und Zerfall der EU beitragen können. Zum anderen haben sie theoretische Ansätze herangezogen, die bisher bei der Analyse europäischer Integration ein Nischendasein gefristet hatten - wie die Theorie der hegemonialen Stabilität ${ }^{16}$ oder historisch-soziologische Analysen der Staatenbildung. ${ }^{17}$

Bisher füllt jedoch der Postfunktionalismus die Leerstellen der älteren Integrationstheorien am systematischsten. Er baut auf Analysen der öffentlichen Meinung und der Parteienentwicklung in der EU auf und entwickelt diese zu einer Theorie der Integrationsdynamik weiter. ${ }^{18}$ Im Gegensatz zur effizienzorientierten, funktionalen Integrationslogik des Intergouvernementalismus und des Neofunktionalismus hebt der Postfunktionalismus ein genuines gesellschaftliches Interesse an der Bewahrung und Stärkung der nationalen Identität und Selbstbestimmung hervor. Droht die europäische Integration diese Identität und

11 Frank Schimmelfennig: Liberal intergovernmentalism and the euro area crisis, in: Journal of European Public Policy 2/2015, S. 177ff.; Frank Schimmelfennig: European integration (theory) in times of crisis. A comparison of the euro and the Schengen crises, in: Journal of European Public Policy 7/2018, S. 969ff.

12 Erik Jones/R. Daniel Kelemen/Sophie Meunier: Failing Forward? The Euro Crisis and the Incomplete Nature of European Integration, in: Comparative Political Studies 7/2016, S. 1010ff. Vgl. Sandra Lavenex: 'Failing Forward' Towards Which Europe? Organized Hypocrisy in the Common European Asylum System, in: Journal of Common Market Studies 5/2018, S. 1195ff.; Marco Scipioni: Failing forward in EU migration policy? EU integration after the 2015 asylum and migration crisis, in: Journal of European Public Policy 9/2018, S. 1357ff.

13 Schimmelfennig: European integration (theory), 2018, S. 979.

14 Schimmelfennig: Liberal Intergovernmentalism and the Crises, 2018, S. 1589.

15 Vgl. aber Zoe Lefkofridi/Philippe C. Schmitter: Transcending or Descending? European Integration in Times of Crisis, in: European Political Science Review 1/2015, S. $3 \mathrm{ff}$.

16 Douglas Webber: European Disintegration? The Politics of Crisis in the European Union, London 2019.

17 Vollaard: European Disintegration, 2018.

18 Hooghe/Marks: Postfunctionalist Theory, 2009. 
Selbstbestimmung zu unterminieren, löst sie einen Prozess der Politisierung aus, durch den die EU zu einem prominenten und umstrittenen Gegenstand der politischen Auseinandersetzung und Mobilisierung in den Mitgliedstaaten wird. ${ }^{19}$ Europäische Integration wird zum festen Bestandteil des nationalen demokratischen Parteienwettbewerbs. Parteien richten ihre Programmatik und Strategie an der integrationspolitischen Konfliktlinie aus und Probleme der Integration werden zum Gegenstand von Volksabstimmungen, Wahlkämpfen und Regierungsbildungen. Vor allem Parteien der radikalen und populistischen Rechten können die Opposition gegen europäische Integration bruchlos mit ihrer nationalistischen, fremdenfeindlichen und antielitären Programmatik vereinbaren. Sie sind daher die größten Profiteure der Politisierung. Die Politisierung der Integration und der Machtzuwachs euroskeptischer Parteien erschweren Integrationsverhandlungen; es droht europapolitische Stagnation, wenn nicht gar die Desintegration der EU. ${ }^{20}$

Der Postfunktionalismus wirft damit Licht auf Krisenphänomene wie das Erstarken des Euroskeptizismus, das Brexit-Votum und die Rechtsstaatlichkeitskrise, die entweder außerhalb des Blickwinkels der traditionellen Integrationstheorien liegen oder diesen rätselhaft erscheinen müssen. Allerdings ist der Postfunktionalismus überwiegend eine Theorie der innenpolitischen Konsequenzen der Integration und der Einschränkung des internationalen und supranationalen (Ver-)Handlungsspielraums. Zum Politikprozess auf der europäischen Ebene und seinen institutionellen und materiellen Ergebnissen hat er wenig zu sagen.

Bei allen Erkenntnissen, welche die Wiederbelebung der traditionellen Integrationstheorien, der Postfunktionalismus und die theoretischen Erkundungen europäischer Desintegration für das Verständnis der EU-Krisen und ihrer Konsequenzen erbracht haben: Die vorherrschende Konzeption von Integration leidet an einem doppelten Bias, das den Blick auf mögliche Integrationsentwicklungen verstellt. Zum einen konzentriert sie sich auf die inneren Grenzen der EU; zum anderen hat sie die Tendenz, die europäische Integration mit einer liberalen politischen und ökonomischen Programmatik zu identifizieren.

Die aktuellen Integrationstheorien sind überwiegend darauf fokussiert, die Entwicklung der inneren Grenzen der EU zu erklären, und sie beziehen sich dabei auf interne Akteure und Beziehungen. Der frühe, realistische Intergouvernementalismus rekurrierte noch stark auf die internationale Umwelt der Gemeinschaft und exogene Einflussfaktoren. ${ }^{21}$ Der Liberale Intergouvernementalismus erklärt hingegen die Öffnung der wirtschaftlichen Binnengrenzen mit innergesellschaftlichen Interessen und mit Verhandlungen unter den Mitgliedstaaten. Geopolitische Ideen und Interessen sowie externe Akteure spielen nur noch eine Nebenrolle. ${ }^{22}$ Der Neofunktionalismus führt den Erfolg regionaler Integration ebenfalls in erster Linie auf günstige innergesellschaftliche Bedingungen (wie Pluralismus) zurück. Er schreibt den Integrationsfortschritt einer Vielfalt von Spillover-Prozessen zu, die mit der kleinen Ausnahme des "geografischen Spillover“ rein endogener Natur sind und die inter-

19 Pieter de Wilde/Michael Zürn: Can the Politicization of European Integration be Reversed?, in: Journal of Common Market Studies s1/2012, S. 137ff.

20 Hooghe/Marks: Postfunctionalist Theory, 2009, S. 21 f.

21 Vgl. u.a. Stanley Hoffmann: Obstinate or Obsolete? The Fate of the Nation-State and the Case of Western Europe, in: Daedalus 3/1966, S. 862ff.

22 Moravcsik: Choice for Europe, 1998, S. 28, 474. 
nen Grenzen zwischen den Mitgliedstaaten inkrementell öffnen. ${ }^{23}$ Erst als Ernst Haas Mitte der 1970er Jahre über die Krise der Integration(-stheorie) schrieb, brachte er die „Externalisierung" - die Interdependenz und Kooperation mit externen Akteuren - als einen desintegrationsförderlichen Prozess ins Spiel. ${ }^{24}$ Schließlich befasst sich auch der Postfunktionalismus mit der mitgliedstaatlichen Politisierung der Integration und mit dem Druck, der von der Politisierung auf die Offenheit und supranationale Kontrolle der Binnengrenzen ausgeht. Trotz gelegentlicher Appelle, exogenen Einflüssen und der geopolitischen Einbettung der Integration stärkere Aufmerksamkeit zu widmen, ${ }^{25}$ hat sich am Binnenfokus der Integrationstheorien wenig geändert. Andererseits bewegt sich die umfassende Literatur, die es zu den Außenbeziehungen und zur externen Grenzpolitik der EU durchaus gibt, ganz überwiegend außerhalb des konzeptionellen und theoretischen Rahmens der Integrationstheorien.

Angesichts der Krisen der EU und der aktuellen geopolitischen Entwicklungen erscheint dieser Binnenfokus allerdings immer weniger plausibel - wenn er es je wirklich war. Die Eurokrise nahm ihren Ausgangspunkt in der US-Hypothekenkrise; die Ursprünge der Flüchtlingskrise liegen in den politischen und wirtschaftlichen Krisen im Vorderen Orient. Bei den Verhandlungen über den Brexit geht es um die Durchlässigkeit und Kontrolle der äußeren Grenzen der EU. Und der Brexit und die Rechtsstaatlichkeitskrise der EU werden durch populistische, nationalistische und autoritäre Regierungen befeuert und unterstützt, die von den USA bis Russland um die EU herum Einzug gehalten haben und die Außengrenzen der EU und ihrer Assoziationspartner infrage stellen. Vor allem in der Flüchtlingsund Brexit-Krise zeigt sich auch, wie der innere Zusammenhalt und die interne Offenheit der EU mit der Definition und Kontrolle der externen Grenzen zusammenhängen.

Das zweite Bias betrifft die Gleichsetzung von europäischer Integration und liberaler Programmatik. Prinzipiell ist Integration, verstanden als Delegation staatlicher Kompetenzen an eine multilaterale regionale Organisation, hinsichtlich der inhaltlichen Politikziele undefiniert. Die Anfänge der europäischen Integration waren - vor allem in der Kohle-, Stahl- und Agrarpolitik - dezidiert nicht marktliberal, sondern dirigistisch. Seit den 1980er Jahren ist europäische Integration hingegen - sowohl in affirmativer als auch in kritischer Perspektive - weitgehend als (neo-)liberales Projekt identifiziert worden. Um nur einige Analysen zu nennen: Bei der Entwicklung des Binnenmarkts überwog die marktöffnende (negative) gegenüber der marktregulierenden (positiven) Integration. ${ }^{26}$ Die Europäische Kommission verwendete die Markt- und Globalisierungslogik strategisch, um das Binnenmarktprojekt voranzubringen, ${ }^{27}$ und die quasikonstitutionelle Kodifizierung der Marktfreiheiten sowie die Rechtsprechung des Europäischen Gerichtshofs erschweren legislative

23 Ernst B. Haas: The Uniting of Europe. Political, Social and Economic Forces 1950-1957, 2. Auflage, Stanford 1968.

24 Ernst B. Haas: Turbulent fields and the theory of regional integration, in: International Organization 2/1976, S. $173 \mathrm{ff}$., hier S. 176.

25 So z.B. Arne Niemann: Explaining Decisions in the European Union, Cambridge 2006, S. 291; Hooghe/Marks: Postfunctionalist Theory, 2009, S. 23.

26 Fritz W. Scharpf: Negative and Positive Integration in the Political Economy of European Welfare States, in: Gary Marks/Fritz W. Scharpf/Philippe Schmitter/Wolfgang Streeck: Governance in the European Union, London u.a. 1996, S. $15 \mathrm{ff}$.

27 Nicolas Jabko: Playing the Market. A Political Strategy for Uniting Europe, 1985-2005, Ithaca 2006. 
Korrekturen. ${ }^{28}$ Die Wirtschafts- und Währungsunion reflektierte einen neoliberalen geldpolitischen Konsens ${ }^{29}$ und neoliberale wirtschaftliche Ideen erwiesen sich auch in der Finanz- und Eurokrise als beharrlich. ${ }^{30}$ Nach außen propagierte die EU ihre liberaldemokratischen Werte und Normen und orientierte ihre Erweiterungsentscheidungen an der liberaldemokratischen Konsolidierung ihrer Nachbarstaaten. ${ }^{31}$ Insgesamt verstand und gebar sich die EU als Avantgarde und Laboratorium einer liberalen Weltordnung. ${ }^{32}$

Angesichts dieser breiten Identifizierung von europäischer Integration und liberaler Programmatik ist es nicht verwunderlich, wenn eine Abkehr von liberalen Inhalten oftmals mit Euroskeptizismus gleichgesetzt wird. Allerdings verstehen sich die Vertreter einer solchen Abkehr - allen voran der ungarische Ministerpräsident Viktor Orbán - dezidiert nicht als Gegner Europas und als Austrittskandidaten, sondern als Verteidiger der „europäischen Zivilisation“ gegen den Multikulturalismus und die „liberale Elite“ sowie als Verfechter einer anderen, „christlichen“ EU. ${ }^{33}$ Außerdem findet sich auch in der Vision eines „schützenden Europas“ bei Emmanuel Macron, dem integrationspolitischen Gegenpol zu Orbán, eine Abkehr vom liberalen Entgrenzungsparadigma - etwa, wenn er in seinem „Brief an die EU-Bürger“ vom März 2019 die „Notwendigkeit schützender Grenzen“ betont und fragt: „Welches Land kann sich allein der aggressiven Strategien der Großmächte erwehren? Wer kann allein seine Unabhängigkeit von den Internetgiganten behaupten? Wie könnten wir ohne den Euro, der die gesamte EU stark macht, den Krisen des Finanzkapitalismus widerstehen?"34 Schon immer hat sich die Integrationsforschung schwergetan, das Konzept des Euroskeptizismus analytisch zu greifen und seine institutionellen und materiellen Komponenten zu trennen. ${ }^{35}$

Angesichts der offensichtlichen Bedeutung der Außengrenzen für die Krisen der EU und der mehr oder weniger deutlichen Korrektur des liberalen Integrationsparadigmas bei den Antipoden des integrationspolitischen Konflikts sind eine Erweiterung und Öffnung der vorherrschenden Konzeption europäischer Integration angezeigt, um die aktuelle Integrationsentwicklung angemessen abbilden und erklären zu können.

\section{Grenzziehung und Integration}

In einem systemischen Verständnis hat Integration zwei Seiten: den inneren Zusammenhalt zwischen den Einheiten eines Systems und die äußere Abgrenzung des Systems

28 Susanne K. Schmidt: The European Court of Justice and the Policy Process. The Shadow of Case Law, Oxford 2018.

29 Kathleen R. McNamara: The Currency of Ideas. Monetary Politics in the European Union, Ithaca 1998.

30 Vivien A. Schmidt/Mark Thatcher (Hrsg.): Resilient Liberalism in Europe's Political Economy, Cambridge 2013.

31 Frank Schimmelfennig: The EU, NATO and the Integration of Europe. Rules and Rhetoric, Cambridge 2003; Frank Schimmelfennig/Stefan Engert/Heiko Knobel: International Socialization in Europe. European Organizations, Political Conditionality and Democratic Change, Basingstoke 2006.

32 Sonia Lucarelli/Ian Manners (Hrsg.): Values and Principles in European Union Foreign Policy, London 2006.

33 Zitiert nach VoxEurop: Viktor Orbáns Europa: „Wir sind die Zukunft Europas“, 11. Januar 2019.

34 Zitiert nach Spiegel Online: Macrons Brief an die EU-Bürger im Wortlaut. „Der Moment des Neubeginns in Europa“, 5. März 2019.

35 Vgl. z.B. Paul Taggart: A Touchstone of Dissent: Euroscepticism in Contemporary Western European Party Systems, in: European Journal of Political Research 3/1998, S. 363ff.; Petr Kopecký/Cas Mudde: The Two Sides of Euroscepticism: Party Positions on European Integration in East Central Europe, in: European Union Politics $3 / 2002$, S. $297 \mathrm{ff}$. 
gegenüber anderen Systemen. Anders gesagt: Integration umfasst die Öffnung und den Abbau der internen Grenzen sowie den Aufbau und die Schließung äußerer Grenzen. Gelungene Integration verknüpft Entgrenzung im Innern mit Eingrenzung gegen außen. Konzeptionen und Analysen der europäischen Integration bleiben daher unvollständig, wenn sie sich ausschließlich oder vorrangig auf die Grenzen zwischen den Mitgliedstaaten fokussieren und Integration und Desintegration an der Öffnung und Schließung der internen Grenzen festmachen. Die Beschreibung und Erklärung der Öffnung und Schließung der äußeren Grenzen der EU und des Wechselspiels zwischen innerer und äußerer Grenzziehung sind unerlässlich, um zu einem umfassenden Verständnis der Entwicklung europäischer Integration zu gelangen.

Folgt man dieser Konzeption, dann lassen sich Integration und Desintegration nicht mehr eindimensional mit Entgrenzung und Eingrenzung gleichsetzen. Entgrenzung und Eingrenzung können sich nicht nur an den inneren und äußeren Grenzen der EU gegenläufig entwickeln - sie müssen es sogar, wenn wir von gelungener Integration sprechen wollen. Aus diesen Überlegungen und der Möglichkeit der internen und externen Entgrenzung und Eingrenzung ergeben sich vier Typen und Dynamiken der Integration (siehe Tabelle 1).

Tabelle 1: Eingrenzung, Entgrenzung und Typen der Integration

\begin{tabular}{|c|c|c|c|}
\hline \multicolumn{2}{|c|}{} & \multicolumn{2}{c|}{ Extern (Außengrenzen) } \\
\cline { 3 - 4 } \multicolumn{2}{|c|}{} & Eingrenzung & Entgrenzung \\
\hline \multirow{2}{*}{$\begin{array}{c}\text { Intern } \\
\text { (Binnengrenzen) }\end{array}$} & Eingrenzung & Defensive Integration & Desintegration \\
\cline { 2 - 4 } & Entgrenzung & Effektive Integration & Dilutive Integration \\
\hline
\end{tabular}

Quelle: Eigene Darstellung.

„Effektive Integration“ ergibt sich demnach aus der Kombination von interner Entgrenzung und externer Eingrenzung. Die Verbindung von sowohl interner als auch externer Eingrenzung, bei der die Mitgliedstaaten zwar gemeinsame Außengrenzen errichten, aber auch die Binnengrenzen untereinander aufrechterhalten oder schließen, lässt sich als „defensive Integration“ etikettieren. Der umgekehrte Fall einer Verbindung von interner und externer Entgrenzung, in dem die Mitgliedstaaten sowohl ihre Binnengrenzen als auch die Außengrenzen der Union öffnen und abbauen, kann mit einem Neologismus als „dilutive“, also sich verdünnende oder auslaufende Integration bezeichnet werden, weil die mangelnde Abgrenzung nach außen die interne Integration gleichsam verwässert. Defensive und dilutive Integration sind demnach Typen der „ineffektiven“ Integration, weil jeweils ein konstitutives Element gelungener Integration fehlt. Der Begriff der „Desintegration“ bleibt dann reserviert für die Kombination aus interner Eingrenzung und externer Entgrenzung. Als Prozess bezeichnet Desintegration die Schließung von Binnengrenzen und die Öffnung oder den Abbau gemeinsamer Außengrenzen. Damit kehren die vormals integrierten Einheiten in den Grundzustand des internationalen Systems zurück.

Die Annahme, dass die Kombination aus interner Entgrenzung und äußerer Eingrenzung „effektiv“ ist, während andere Kombinationen zu Integrationsdefiziten führen, ergibt sich nicht nur aus dem systemtheoretischen Integrationsbegriff, sondern auch aus Rokkans mittlerweile klassischer Analyse des modernen europäischen Staatenbildungsprozesses als 
Interaktion von externer Grenzziehung und interner politischer Strukturierung. ${ }^{36} \mathrm{Um}$ diesen Prozess zu erklären, bedient sich dieser Albert Hirschmans Unterscheidung von Abwanderung („Exit“), Widerspruch („Voice“) und Loyalität als Reaktionen auf Leistungsabfall von Organisationen. ${ }^{37}$ Während Abwanderung die typische Reaktion von Firmenkunden in einem funktionierenden Markt ist, ist Widerspruch charakteristisch für Organisationen (wie die Familie oder den Staat), die dem Individuum keine oder keine attraktiven Abwanderungsmöglichkeiten bieten. In auf freiwilliger Mitgliedschaft beruhenden Vereinen oder Verbänden - und dazu zählt auch die EU - finden sich beide Reaktionsweisen. In dem Maße, wie die Hindernisse für den „Exit“ zunehmen und/oder die Kosten und Hürden für interne Veränderungen abnehmen, orientieren sich die Mitglieder solcher Vereinigungen von der Abwanderung zum Widerspruch um. Analog dazu sieht Rokkan die zunehmende Schließung und Kontrolle der Außengrenzen europäischer Territorien als eine zentrale Voraussetzung für die Entwicklung des demokratischen National- und Wohlfahrtsstaates. ${ }^{38}$

Bartolini formuliert den Zusammenhang von externer Grenzziehung und interner politischer Strukturierung nicht nur weiter aus, sondern überträgt die Rokkansche Argumentation auch auf die europäische Integration als jüngste Phase des politischen Entwicklungsprozesses Europas. ${ }^{39}$ Die Kontrolle und Schließung der externen territorialen Grenzen durch das politische Zentrum (den Staat oder die EU) hindern Akteure und Ressourcen an der Abwanderung und binden sie an das Territorium. Damit stärkt, erstens, das Zentrum die kollektive Identität, Vertrauensbildung und Verhaltenskonformität der Binnenakteure. Zweitens wird verhindert, dass Akteure sich durch Abwanderung ihren öffentlichen Verpflichtungen und der innergesellschaftlichen Solidarität entziehen und die dafür notwendigen Ressourcen außer Landes bringen. Drittens erzeugen die fehlenden Abwanderungsmöglichkeiten aber Nachfrage nach politischer Beteiligung und Mitbestimmung. Die Schließung der externen Grenzen fördert somit die „politische Produktion“ (von öffentlichen Gütern und Solidarleistungen) und die „politische Strukturierung“ (gesellschaftlicher Konflikte und der Teilhabe am politischen Entscheidungsprozess) - kurz: den demokratischen Wohlfahrts- und Interventionsstaat. ${ }^{40}$ Offene Grenzen und die Ab- und Zuwanderungsmöglichkeiten, die sie schaffen, führen hingegen zur Abnahme politischer Produktion (durch Ressourcenverknappung und Trittbrettfahren) und zu politischer Destrukturierung. ${ }^{41}$

Folgt man dieser Argumentation konsequent, so ist es allerdings unverständlich, warum die europäischen Staaten sich jemals auf die europäische Integration eingelassen haben. Hier kommen wiederum die traditionellen, „funktionalen“ Integrationstheorien ins Spiel: Sie erklären die Nachfrage nach Integration mit den politischen und wirtschaftlichen Stabi-

36 Stein Rokkan: Staat, Nation und Demokratie in Europa. Die Theorie Stein Rokkans aus seinen gesammelten Werken rekonstruiert und eingeleitet von Peter Flora, Frankfurt am Main 2000.

37 Albert O. Hirschman: Abwanderung und Widerspruch. Reaktion auf Leistungsabfall bei Unternehmungen, Organisation und Staaten, Tübingen 2004.

38 Stein Rokkan: Entries, voices, exits: Towards a possible generalization of the Hirschman model, in: Social Science Information 1/1974, S. 39ff., hier S. 49.

39 Stefano Bartolini: Restructuring Europe. Centre formation, system building, and political structuring between the nation state and the European Union, Oxford 2005.

40 Ebenda, S. 40ff.

41 Ebenda, S. 53. 
litäts- und Effizienzgewinnen, die kleinräumige staatliche Einheiten durch die Öffnung ihrer Grenzen, gemeinsame Märkte und internationale Institutionen erzielen können. In der theoretischen Perspektive von Rokkan und Bartolini geht eine einseitig durch Entgrenzung auf Effizienzgewinne orientierte Integration allerdings auf Kosten (nationaler) Identität, Solidarität und Demokratie. ${ }^{42}$

Diese Diagnose deckt sich sowohl mit der akademischen Kritik an der (neo-)liberalen Ausrichtung der europäischen Integration als auch mit der postfunktionalistischen Analyse der Politisierung der Integration, der Mobilisierung der wirtschaftlichen und kulturellen Integrationsverlierer und der Restrukturierung der parteipolitischen Konfliktlinien. In der Tat sind zahlreiche postfunktionalistische Argumente und Erwartungen bereits bei Bartolini angelegt. Rokkan und Bartolini weisen jedoch auf einen Integrationsweg hin, der prinzipiell geeignet ist, die liberale Entgrenzung der EU auszubalancieren und ihren integrationsgefährdenden Tendenzen entgegenzuwirken, ohne jedoch die Skalengewinne der internen Entgrenzung infrage zu stellen: die externe Eingrenzung.

Als Zwischenfazit gilt es festzuhalten, dass eine erweiterte, sowohl die internen als auch die externen Grenzen einbeziehende Integrationskonzeption nicht nur zu einer vollständigeren Beschreibung des Integrationsprozesses und seiner Entwicklungsrichtungen führt, sondern auch auf eine erweiterte Diagnose und alternative Therapie der Integrationskrisen verweist. Demnach resultierten die Krisen der EU nicht nur, aber auch aus einer dilutiven Integrationsdynamik, bei der die (neo-)liberale interne und externe Öffnung der EU Hand in Hand gingen und beide nicht hinreichend durch externe Grenzkontrollkapazitäten und interne Kompensationskapazitäten abgesichert wurden. Entsprechend eröffnen die (partielle) Schließung der Außengrenzen und der Aufbau externer Grenzkontrollkapazitäten eine Alternative zur europäischen Desintegration, die entweder zu einer defensiven Integration führen oder - im aus integrationstheoretischer Sicht günstigsten Fall - die EU sogar auf den Pfad effektiver Integration (zurück-)bringen könnte. Der folgende grobe Überblick über die jüngere Integrationsentwicklung soll zeigen, dass ein solcher Wandel von der externen Entgrenzung zur Eingrenzung als Reaktion auf die Politisierung und die Krisen der EU in der Tat zu beobachten ist.

\section{Von der Entgrenzung zur Eingrenzung}

In einem institutionellen Verständnis bestehen Grenzen aus einem Regelwerk. Es legt fest, wo die Grenze zwischen innen und außen verläuft, wie durchlässig sie für Transaktionen zwischen innen und außen ist, über welche Kompetenzen und Kapazitäten das Zentrum für die Kontrolle der Grenzen verfügt und wie kongruent das Grenzregime in unterschiedlichen Politikfeldern ist. In der EU zeigt sich Entgrenzung in der Erweiterung der Mitgliedschaft, der Öffnung von Grenzen, dem Abbau von Grenzkontrollen und in differenzierter Integration - also der politikfeldspezifischen Variabilität von Grenzverläufen und -regimes. Eingrenzung besteht entsprechend in der Konsolidierung (oder gar Verringerung) der Mitgliedschaft, in höheren regulatorischen Hürden und verstärkten Kontrollen grenzüberschreitender Transaktionen sowie in der Entdifferenzierung der Integration also einer höheren Kongruenz der politikfeldspezifischen Grenzregimes.

42 Ebenda, S. 242ff. 
Demnach war die Integrationsphase seit Mitte der 1980er Jahre von einer weitreichenden Entgrenzungsdynamik geprägt, die von externen wie internen Faktoren ausgelöst wurde. Externe Faktoren waren in erster Linie die „dritte Demokratisierungswelle“43, die zunächst Südeuropa und dann Osteuropa ergriff und die Erweiterung der EU vorantrieb, sowie die einsetzende Globalisierung, die zu einer globalen Integration von Produktionsketten und einer Liberalisierung des Welthandels führte. Der sich verschärfende internationale Wettbewerb übte zudem Druck auf die Gemeinschaft aus, die „Eurosklerose“ der 1970er Jahre zu überwinden; und die Konvergenz der wirtschaftlichen Präferenzen der wichtigsten Mitgliedstaaten ermöglichte es diesen, sich auf eine liberale Marktöffnungsagenda zu einigen. ${ }^{44}$

Der Binnenmarkt, die Wirtschafts- und Währungsunion und das Schengen-Regime waren die zentralen internen Entgrenzungsprojekte dieser Phase. Sie wurden aber nicht von einer entsprechenden externen Eingrenzung begleitet oder durch sie kompensiert. Vielmehr gingen interne und externe Entgrenzung Hand in Hand. Parallel zur Vertiefung des Binnenmarkts schloss sich die EU der Reform des Allgemeinen Zoll- und Handelsabkommens (General Agreement on Tariffs and Trade, GATT) zur Liberalisierung des Welthandels und zur Gründung der Welthandelsorganisation an. Der Abbau von Kapitalverkehrskontrollen und die Integration der Finanzmärkte fanden sowohl in der EU als auch global statt. Das Schengen-Regime war nicht in gleicher Weise in eine globale Liberalisierung eingebunden, ${ }^{45}$ doch ging die Globalisierung auch mit einem Anstieg der internationalen Migration einher. Schließlich erweiterte und differenzierte sich die EU in dieser Phase in einem bisher ungekannten Ausmaß. Seit 1980 stieg die Anzahl der Mitgliedstaaten der EU von neun auf 28. Für diejenigen Staaten, die der EU, oder jedenfalls der Währungsunion oder dem Schengen-Regime, nicht als Vollmitglieder beitreten wollten oder konnten, schuf die EU ein Netz von politikfeldspezifischen internen und externen Differenzierungen von der Eurozone und dem Schengen-Raum über die Binnenmarktintegration im Europäischen Wirtschaftsraum, die bilateralen Verträge mit der Schweiz und die Zollunion mit der Türkei bis hin zu Assoziations- und Freihandelsverträgen mit den Staaten der Östlichen Partnerschaft. ${ }^{46}$

In der Kontrolldimension zeigte sich die Entgrenzungsdynamik schließlich in einem Ungleichgewicht zwischen dem Abbau nationaler und dem Aufbau supranationaler Kompetenzen und Kapazitäten. Nationale Behörden, die traditionell über hohe Kapazitäten der Grenzkontrolle verfügten, wurden in ihrer Autonomie weitgehend eingeschränkt. Und während die Mitgliedstaaten den supranationalen Behörden - etwa der Europäischen Kommission oder dem Europäischen Gerichtshof - starke regulatorische Kompetenzen bei der Durchsetzung der Grenzöffnung gegenüber den Mitgliedstaaten verliehen, waren sie

43 Samuel P. Huntington: The Third Wave: Democratization in the Late Twentieth Century, Norman 1991.

44 Geoffrey Garrett: International cooperation and institutional choice: the European Community's internal market, in: International Organization 2/1992, S. 533ff.; N. Piers Ludlow: From Deadlock to Dynamism. The European Community in the 1980s, in: Desmond Dinan (Hrsg.): Origins and Evolution of the European Union, Oxford 2006, S. 217ff.; Andrew Moravcsik: Negotiating the Single European Act: national interests and conventional statecraft in the European Community, in: International Organization 1/1991, S. 19ff.

45 Alexander Betts (Hrsg.): Global Migration Governance, Oxford 2011.

46 Frank Schimmelfennig/Thomas Winzen: Ever Looser Union? Differentiated European Integration, Oxford, im Erscheinen. 
zurückhaltend damit, europäische Organe mit unabhängigen und nennenswerten fiskalischen, administrativen oder Zwangskapazitäten auszustatten.

Dieses Ungleichgewicht gilt sowohl für die Dominanz der negativen gegenüber der positiven Integration im Binnenmark $\mathrm{t}^{47}$ als auch für die Integration staatlicher Kernkompetenzen wie innerer und äußerer Sicherheit oder die wohlfahrtsstaatliche Daseinsvorsorge. ${ }^{48} \mathrm{Im}$ Schengen-Raum wurden nationale interne Grenzinfrastrukturen und -kontrollen abgebaut und die Kontrolle der EU-Außengrenzen den Mitgliedstaaten übertragen, während die Harmonisierung der Asyl- und Einwanderungsregeln und der Aufbau europäischer Grenzsicherungskapazitäten (wie Frontex) nur schleppend vorangingen. ${ }^{49}$ Die Regeln der Währungsunion verwehrten es den Staaten, auf Wirtschaftskrisen durch Währungsabwertung oder (hohe) Schuldenaufnahme zu reagieren, ohne dass die Eurozone selbst über das Mandat oder die fiskalischen Kapazitäten verfügte, den Mitgliedstaaten durch Transfers oder Risikoteilung zu Hilfe zu kommen.

Insgesamt mündete die dynamische Entwicklung der europäischen Integration also in eine dilutive Integration: in eine breit angelegte interne Entgrenzung der EU, die nicht durch eine entsprechende externe Eingrenzung kompensiert und stabilisiert wurde, sondern durch die wirtschaftliche Öffnung, die Erweiterung und die Differenzierung der EUAußengrenzen sogar noch weiter verstärkt wurde. ${ }^{50}$ Der dilutive Integrationsmodus sorgte für wirtschaftliche Dynamik in Europa und stärkte individuelle Freiheiten und Chancen. Er versäumte es jedoch, die EU mit den notwendigen Kompetenzen und Kapazitäten auszustatten, um interne Ungleichgewichte zu kompensieren und externen Schocks wirksam entgegenzutreten.

Die negativen Folgen dieser überschießenden Entgrenzung zeigten sich in der „Polykrise“ der EU. Zum einen verstärkten die Erweiterung und Öffnung der EU-Außengrenzen die Wirkung exogener Schocks. Durch die Verflechtung der Finanzmärkte zog die amerikanische Hypothekenkrise europäische Banken und mittelbar europäische Staatshaushalte in Mitleidenschaft. Die profitable globale Handelsverflechtung macht Europa zugleich verwundbar gegenüber dem amerikanischen und chinesischen Merkantilismus und den sich verschärfenden Handelskonflikten zwischen den beiden. Durch die Erweiterung und externe Differenzierung konnte die EU zwar ihr unmittelbares Umfeld befrieden und demokratisieren. Zugleich rückte sie jedoch näher an die Krisenherde im Nahen Osten heran und geriet in Konflikt mit dem zunehmend aggressiven Nationalismus Russlands; und indem die EU junge Demokratien aufnahm, importierte sie auch deren rechtsstaatliche Schwächen und Defizite.

Zum anderen verfügte die EU nicht über effektive Kompetenzen und Kapazitäten zur Abfederung und Kompensation dieser Schocks, weshalb sich externe Schocks zu internen Integrationskrisen entwickelten. Es fehlte der Eurozone an Regeln und Mechanismen zur Rettung oder Insolvenz von Banken und Staaten. Dem Schengen-/Dublin-Regime mangelte es an Kapazitäten für die effektive Kontrolle der Außengrenzen und an einer die Mittel-

47 Scharpf: Negative and Positive Integration, 1996.

48 Philipp Genschel/Markus Jachtenfuchs (Hrsg.): Beyond the Regulatory Polity? The European Integration of Core State Powers, Oxford 2014.

49 Vgl. u.a. Sandra Lavenex: Mutual recognition and the monopoly of force: limits of the single market analogy, in: Journal of European Public Policy 5/2007, S. 762ff.

50 Vgl. Bartolini: Restructuring Europe, 2005, S. $177 \mathrm{ff}$. 
meeranrainer entlastenden und fairen Verteilung von Flüchtlingen. Die Bestimmungen des Art. 7 EUV haben sich als Antwort auf die Rechtsstaatlichkeitskrise als zahnlos erwiesen. Und die EU ist nicht in der Lage, Mitgliedstaaten, geschweige denn assoziierte Staaten, bei militärischen Bedrohungen zu unterstützen.

Ebenso wie die Entscheidung zur internen und externen Entgrenzung in den 1980er Jahren resultierte auch die Abkehr von der dilutiven Integration aus dem Zusammenspiel EU-interner und internationaler Entwicklungen. War es in den 1980er Jahren die Kombination aus „Eurosklerose“ und wirtschaftsliberaler Konvergenz in einem von der Abschwächung des Kalten Krieges und der aufziehenden Globalisierung und Demokratisierung geprägten internationalen Rahmen, so ist es nun die Kombination aus EU-Krisen und rechtspopulistischer Politisierung in einem Umfeld, das von wirtschaftlicher Entglobalisierung, ${ }^{51}$ der „dritten Welle der Autokratisierung “52 und verschärften Großmachtkonflikten gekennzeichnet ist. Gemeinsam erzeugen diese Entwicklungen einen Eingrenzungsdruck, der prinzipiell in eine defensive europäische Integration oder gar in die Desintegration münden könnte. Bisher überwiegen jedoch die Anzeichen für eine externe Eingrenzung bei fortbestehender interner Entgrenzung - und damit für eine Kurskorrektur zugunsten einer effektiveren Integration.

Die Krise des Welthandelsregimes, das Scheitern der Transatlantischen Handels- und Investitionspartnerschaft (Transatlantic Trade and Investment Partnership, TTIP) und der russische Widerstand gegen die Ostverschiebung der EU-Handelsgrenzen sorgen dafür, dass sich die wirtschaftlichen Außengrenzen der EU konsolidieren. Stattdessen konzentriert sich die EU auf die Verhinderung amerikanischer Handelssanktionen, die Einhegung der amerikanischen Tech-Giganten, die Aufrechterhaltung von Wirtschaftssanktionen gegen Russland und eine härtere, gemeinsame Gangart gegenüber China, das von der Europäischen Kommission im März 2019 erstmals als „Systemrivale“53 gebrandmarkt wurde. Als Reaktion auf die Flüchtlingskrise verschärften die Mitgliedstaaten ihre Asylgesetzgebung, während die EU mit der Umwandlung von Frontex in eine Europäische Grenzund Küstenwache (hohe Mittelzuwächse und die geplante Aufstockung des Personals auf 10.000 BeamtInnen) massiv in die Grenzkontrollkapazität investierte. Zugleich ist eine Welle an verteidigungspolitischen Initiativen von der Ständigen Strukturierten Zusammenarbeit über die Europäische Interventionsinitiative bis hin zu Plänen für einen Europäischen Sicherheitsrat zu beobachten.

Eine Mischung aus negativer öffentlicher Meinung sowie populistischer Mobilisierung in den Mitgliedstaaten und Stagnation oder Rückschritten bei der demokratischen Konsolidierung in den Kandidatenstaaten hat dazu geführt, dass sich der Erweiterungsprozess erheblich verlangsamt hat. Seit der Aufnahme Kroatiens 2013 ist kein weiterer Staat auch nur in die Nähe eines baldigen Beitritts gerückt. Verhandlungen mit der Türkei finden nur noch auf dem Papier statt und eine Beitrittsperspektive für die Staaten der Östlichen Partnerschaft ist nicht zu erwarten. Stattdessen hat mit dem Vereinigten Königreich ein Mitgliedstaat seinen Austritt aus der EU verhandelt.

51 The Economist: Globalisation has faltered. It is now being reshaped, 24. Januar 2019.

52 Anna Lührmann/Staffan I. Lindberg: A third wave of autocratization is here: what is new about it?, in: Democratization 7/2019, S. 1095ff.

53 Europäische Kommission/Hohe Vertreterin der Union für Außen- und Sicherheitspolitik: EU-China - Strategische Perspektiven, JOIN(2019) 5 final. 
Wenn das Vereinigte Königreich die EU verlässt, wird sich auch die interne Differenzierung der EU deutlich verringern. Möglicherweise erhöht sich dadurch auch der Druck auf kleinere Mitgliedstaaten, ihre Opt-outs von der Währungsunion, der Bankenunion und der innenpolitischen Integration zu überdenken. In den Brexit-Verhandlungen hat die EU das Prinzip der Unteilbarkeit des Binnenmarkts gestärkt und damit der vom Vereinigten Königreich gewünschten, flexiblen externen Differenzierung eine Absage erteilt. In diesem Zusammenhang drängt die EU auch andere Teilnehmer an der externen Differenzierung beispielsweise die Schweiz - zu einer stärkeren regulatorischen Angleichung und institutionellen Kontrolle.

Im Gegensatz dazu sind die Binnengrenzen von hoher Stabilität der Entgrenzung und in der Eurozone - sogar von stärkerer Integration gekennzeichnet. Nur im SchengenRaum hat seit 2015 im Zuge der Wiedereinführung von internen Grenzkontrollen durch eine Handvoll Staaten eine gewisse interne Eingrenzung stattgefunden. In der Priorisierung der externen gegenüber der internen Eingrenzung treffen sich die Präferenzen der rechtspopulistischen und der zentristischen Parteien und Regierungen. Für die selbst ernannten Verteidiger des „christlichen Europa“ hat die Abwehr von EinwanderInnen aus anderen Kulturen Vorrang; für die des „liberalen Europa“ sind die Kontrolle und Schließung der Außengrenzen ein relativ kostengünstiges Zugeständnis an ihre populistischen Kritiker, um Desintegrationsforderungen die Spitze zu nehmen und Zustimmung für die weitaus bedeutendere Offenheit der Binnengrenzen zu erzielen.

\section{Eingrenzung als Mittel der Konsolidierung?}

Ich habe in diesem Beitrag argumentiert, dass die Integrationsforschung von einer zweifachen Erweiterung des vorherrschenden Integrationskonzepts profitieren würde. Anstatt sich auf die Integration der Binnengrenzen zu fokussieren, sollte sie die Integration der Außengrenzen und ihr Zusammenspiel mit der Binnenintegration gleichgewichtig in den Blick nehmen. Anstatt die europäische Integration mit einer liberalen wirtschaftlichen und politischen Programmatik zu identifizieren, sollte sie alternative Politikinhalte als prinzipiell integrationskompatibel einbeziehen. In der Tat ist das vorherrschende liberale Entgrenzungskonzept der Integration perfekt auf die Periode von der Mitte der 1980er Jahre bis zum Ausbruch der jüngsten Integrationskrisen zugeschnitten. Für die Integrationsphase seit der „Polykrise“ erweist es sich aber als zu eng und zu „pessimistisch“.

Das hier skizzierte erweiterte Integrationskonzept weist darauf hin, dass die Krisen des vergangenen Jahrzehnts vor allem auf einen spezifischen Integrationstyp zurückzuführen sind: die aus der Kombination von interner und externer Entgrenzung resultierende dilutive Integration. Die Krise und schrittweise Ablösung dieses Integrationstyps sind daher nicht mit Desintegration gleichzusetzen. Vielmehr hat die EU auf die internen Krisen und die Krise der liberalen Weltordnung in ihrem Umfeld überwiegend mit Schritten der externen Eingrenzung reagiert, während die Integration der Binnengrenzen weitgehend stabil geblieben ist. Dabei hat sie sich unter dem Druck rechtspopulistischer Wahlerfolge zudem von einer liberalen externen Grenzpolitik entfernt. ${ }^{54}$ Das zeigt sich vor allem in der Asylpolitik in rigideren Asylregeln und der externen Kooperation mit autoritären Regimes in

54 Vgl. Lavenex: 'Failing Forward', 2018. 
Nordafrika und in der Türkei, um Flüchtlinge von den europäischen Grenzen fernzuhalten. Es zeigt sich darüber hinaus auch in einer insgesamt stärker von geopolitischen Interessen geleiteten Nachbarschaftspolitik der EU. ${ }^{55}$

Folgt man Rokkan und Bartolini, so kann eine solche externe Eingrenzungspolitik durchaus dazu beitragen, die Entgrenzungsdefizite zu beheben und die europäische Integration insgesamt zu konsolidieren. Noch ist diese Politik zu neu, um zu beurteilen, wie weit die Schließung der externen Grenzen tatsächlich gehen wird und ob es der EU gelingen wird, eine nennenswerte externe Grenzkontrollkapazität aufzubauen - und damit die interne politische Zustimmung und Strukturierung nachhaltig zu verändern. Jedenfalls ist es der EU gelungen, gegenüber externen Herausforderungen - etwa bei den RusslandSanktionen, den Brexit-Verhandlungen und der Externalisierung des Flüchtlingsproblems - eine hohe Geschlossenheit zu erzielen. Zudem hat das britische Exit-Votum in der öffentlichen Meinung der EU-27 zu einer deutlichen Zunahme der Unterstützung für die EU geführt. ${ }^{56}$ Ebenso bemerkenswert ist, dass zahlreiche extreme Parteien - von Marine Le Pens Rassemblement National über die Schwedendemokraten und die schwedische Linkspartei bis hin zur Alternative für Deutschland - ihre Programme und Strategien von „Exit“ zu „Voice“ umorientieren.

Sollte diese Entwicklung fortschreiten, so wäre es eine veritable „List der Geschichte“, dass gerade illiberale Kräfte innerhalb und außerhalb der EU eine Kurskorrektur zugunsten der externen Eingrenzung erzwungen haben, die sich mittelfristig als Stärkung der europäischen Integration erweisen könnte.

55 Vgl. Cristian Nitoiu/Monika Sus: Introduction: The Rise of Geopolitics in the EU's Approach in its Eastern Neighbourhood, in: Geopolitics 1/2019, S. 1ff.

56 Catherine de Vries: Benchmarking Brexit: How the British Decision to Leave Shapes EU Public Opinion, in: Journal of Common Market Studies S1/2017, S. 38ff. 